\title{
DETERMINATION OF ALUMINUM ROLLING OIL AND MACHINERY OIL RESIDUES ON FINISHED ALUMINUM SHEET AND FOIL USING ELEMENTAL ANALYSIS AND FOURIER TRANSFORM INFRARED SPECTROSCOPY COUPLED WITH MULTIVARIATE CALIBRATION
}

\author{
Özlem İnanç Uçar ${ }^{1}$, Hatice Mollaoğlu Altuner ${ }^{1}$, Mert Günyüz ${ }^{1}$, Mustafa Murat Dündar ${ }^{1}$, Durmuş Özdemir ${ }^{2}$ \\ ${ }^{1}$ Assan Alüminyum San. ve Tic. AS; Tuzla, Istanbul 34940 Turkey \\ ${ }^{2}$ İzmir Institute of Technology; Urla, İzmir 35430 Turkey
}

Keywords: aluminum rolling, rolling oil additives and contaminants, elemental analysis, FTIR, multivariate calibration, genetic algorithms

\begin{abstract}
The surface characteristics of rolled aluminum products such as sheets and foils are strongly affected by the particular rolling process and the type of aluminum rolling oil compositions. After the rolling process, coiled aluminum sheets and foils undergoes annealing to form desired crystal structure and remove the rolling oil residues. Depending on the time and the temperature that rolled aluminum exposed for annealing, rolling oil residues are mostly removed from the coiled aluminum products but if there is any contamination in rolling oil due to hydraulic and gearing parts of the rolling systems these heavier oils are not easily evaporates from the aluminum surfaces especially inner parts of the coiled aluminum sheets and foils. These rolling oil contaminants create serious problems for the some specific applications of these aluminum products in certain industries such as automotive and coating as remaining thin oil layer prevents proper painting and coating. Therefore, it is very crucial for the rolling industry to be able to monitor the heavy oil contamination on the rolled products and determine the source of these contaminants .In this study, it was aimed to develop a nondestructive infrared spectroscopic method combined with chemometric multivariate calibration techniques for the quantitative determination of rolling oil residues and contaminants on the rolled aluminum products. To be able to generate multivariate calibration methods, an industrial elemental analysis system was adopted for the quantitative determination of heavy oil contaminants on the rolled aluminum products and these were used as reference values for infrared analysis of the same samples. In addition, apart from conventional use of elemental analysis systems for the total organic analysis, the raw data (raw chromatogram) obtained from elemental analysis was used to directly generate multivariate calibration models for each contaminant by using synthetically contaminated surfaces as the calibration samples. The results promised that elemental analysis can be used not just for the total organic content but also specifically to determine amount of each contaminant on the aluminum surfaces. İt is also, expected that infrared spectroscopy with grazing angle spectra collection accessories can be used for nondestructive analysis of these contaminants.
\end{abstract}

\section{Introduction}

Flat rolled aluminum production is a rolling process to reduce the thickness with a further objective of improving the strength, surface finish and formability of the stock by conducting the process of rolling at the room temperature. Since the material is subjected to high compression and surface shear stresses, the temperature of the strip can reach the temperature of as high as $70-80^{\circ} \mathrm{C}$ in a particular or consecutive rolling passes. High temperature generated does not only influence the strip itself but also tends to raise the temperature of the rolls and other systems in the vicinity. Large variations beyond certain limits, during the deformation process, also may render the product unsuitable or even lead to the phenomenon of pick up and sticking. It is, therefore, necessary that the heat of deformation is carried away from the system and a thermal stability with pre-defined system temperature is maintained. It is also necessary that required lubricity be provide to eliminate any chance of sticking or any impairment to the aesthetic appearance of the surface of the product. Thus, the main characteristic of the medium need to be used to carry out the function of heat removal is primarily its being a good carrier of heat. Other important features and properties that the medium should possess are to provide required lubricity, maintain its film strength and thickness under high loads exerted by the rolls. While the base oil provides the required heat removal function, additives are effective for maintaining the rolling oil strength at the contact area. Additives are polar or chemically active compounds. By virtue of the polarity, additives are preferentially adsorbed to the metal surface and form a thin lubricating film. Base oil provides the carrier for these reduction additives.

Other mechanical and hydraulic components of the mill, such as hydraulic pistons, bearings etc., employ other type of oils different in chemistry and physical properties than that of base oil and additives. Due to their functionality in those systems and performance expected, they have longer chain length molecules and therefore they are more viscous fluids. Although rolling oil circulates within a closed loop in the mill, mechanical failures in the joints of these components, leakages during maintenance and other reasons introduce them into the closed loop of rolling oil. Rolling oil contaminated with long chain molecule mill lubes is in tendency of creating staining after further annealing operations. With increasing magnitude of contamination, the micromechanism of friction between strip and roll change from boundary film lubrication to hydro-dynamic lubrication mode. Viscosity, rolling speed and pressure are three components of Streibeck curve determining the coefficient of friction and oil film thickness. Hence, any variation in the viscosity of the lubricant directly influences the surface quality of the strip, for example brightness. Extreme case of failure in impairment of the film thickness results in much severe surface defects, such as herring bone.

Degreasing is one of the essential operations for many sheet and coil products. It is conducted by using acidic or alkaline chemicals to clean the residual oil from previous rolling passes. Efficiency of degreasing depends on the amount of residual oil on the material surface, content of residual oil, including long chain molecule oils, type of chemical used and line speed. Materials produced with twin roll casting technology have some additional 
features on the sheet surface that are interpreted as entities needed to be cleaned during degreasing. That is called smut. They are very fine aluminum particles generated by the rolling passes. Regardless of the production method of aluminum products, smut is the unavoidable consequence of rolling operation; however its magnitude is much higher on Twin roll cast materials than its DC cast-hot rolled counterparts. Products involving backannealing operation as the last step of their thermomechanical processing route are assumed to be almost free of rolling oil residue on the surface. But this might not be case if rolling oil is contaminated with viscous long chain mill lubes. While annealing temperature is mostly high enough to evaporate base oil and additive, those contaminants in the base oil with higher initial boiling point might still keep their existence on the surface. As the presence of smut combines with contaminated residual rolling oil on the material surface, efficiency of the degreasing operation decreases. Hence, performance of degreasing operation and resulting surface cleanness is determined primarily by the rolling oil and its composition.

Modern instrumental techniques are offering fast analysis that can generate huge amount of raw data in a short period of time for multicomponent samples. However univariate calibration techniques fail to give the efficient results for those types of data as the components of the mixtures generally produce severely overlapping signals. Cases like this often requires a multivariate calibration approach in which instrumental responses measured on multiple channels related to a chemical or physical property of a sample even though it contains multiple components. In the last couple of decades, chemometrics and advanced computer technology resulted in the development of several multivariate calibration techniques [1-5]. Inverse Least Squares (ILS) is a multivariate calibration method based on inverse Beer's Law in which the concentration of an analysis is modeled as a function of absorbance. On the other hand, full spectral information collected from a spectroscopic technique produces hundreds of data points if not thousands for a given samples and often this spectrum contains many regions with colinearities and some amount of noise. In addition, it also contains absorbance regions where the signal is not exactly linearly related to the concentration of the component being modeled. In these cases, ILS may not offer efficient solutions for a given problem with whole spectral information and therefore, it might be necessary to apply a variable selection before the modeling step. Among several methods of variable selection, Genetic Algorithms (GA) is offering fast and efficient solutions for a given problem [6-11]. Genetic Inverse Least Squares (GILS) is a modified version of ILS method in which a small set of wavelengths is selected from a full spectral data matrix and evolved to an optimum solution using a GA. The detailed description of GILS algorithm has been given in a number of reports elsewhere [12-14] and therefore algorithmic details will not be repeated here.

Previous studies [1] aiming to determine quantities of heavy oil contaminants in the rolling oil by employing FTIR spectroscopic methods provided very reliable and reproducible results. Genetic inverse least square (GILS) method was used as the multivariate calibration and wavelength selection method for each component of the rolling oil. Due to the volume of liquid samples used in this technique provide sufficient information in the spectra to be analyzed in GILS method. However, present study focuses on the quantitative characterization of residual rolling oil on the sheet surface which has very limited in quantity compared to liquid samples. A commercially available industry scale carbonhydrogen analyzer was used to characterize the residual oil on the surface. If each component and contaminant in the rolling oil exhibits different behavior, as they react with oxygen in the chamber of the equipment by gradual change in temperature, each one would generate distinctive chromatograms. Present study aims to exploit this operational principal of the carbon-hydrogen analyzer to quantify the minute amount of residual rolling oil on the material surface.

\section{Experimental}

In order to evaluate the possibility of using carbon - hydrogen elemental analysis system along with FTIR spectroscopy for quantitative determination of residual rolling oil contaminants on the aluminum sheet surfaces, individual heavy oils and rolling oil components were analyzed first by carbon - hydrogen elemental analysis system (LECO 628, LECO Corporation St. Joseph, Michigan USA). Approximately $2.5 \mathrm{mg}$ oil sample were dropped on a $1.0 \times 1.0 \mathrm{~cm}$ piece of aluminum sheet and a heating ramp given in Figure 1 was established which provides partial separation of component peaks in a reasonable time. A total of 25 synthetic mixture were prepared by mixing rolling oil (base oil) Linpar, additive Nafol and four heavy oil namely Hidrotex, Recompound 220, Recompound 320 and Mobil 630. Table 1 shows the concentration profile of the 25 synthetic samples in which the concentration range of the heavy oils were decided on the basis of the results obtained from the previous studies about the rolling oil composition of the rolling mill systems. Same procedure for the elemental analysis was carried out for the synthetic samples as the pure components given above. Elemental analysis chromatograms were recorded for about 1200 second with the increments of 0.1 second and each chromatogram were analyzed in terms of total carbon and hydrogen with two $\mathrm{CO}_{2}$ detectors $\left(\mathrm{CO}_{2}\right.$ low and $\mathrm{CO}_{2}$ high $)$ and one $\mathrm{H}_{2} \mathrm{O}$ detector. The chromatograms were saved as ascii text file and transferred to the computer where the multivariate calibration models were developed based on the $\mathrm{CO}_{2}$ low detector chromatograms. In addition to the synthetic samples and the pure components, two real aluminum sheet samples were also analyzed with the triplicate samples taken from rolled aluminum sheet from right edge, middle and left edge of each.

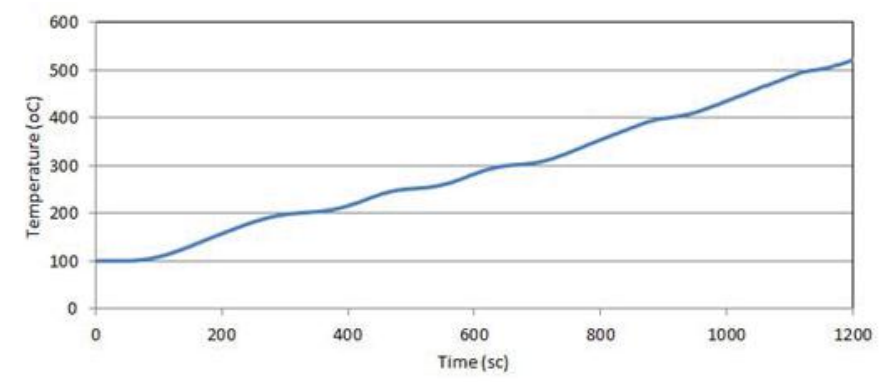

Figure 1. Heating ramp used in elemental analysis. 
Table 1. Concentration profile of the synthetic samples. The concentration unit for each component is given as weight percentage $(\mathrm{w} / \mathrm{w} \%)$.

\begin{tabular}{|l|r|r|r|r|r|r|}
\hline No & Mobil 630 & Recompound 220 & Recompound 320 & Hidrotex 46 & \multicolumn{1}{l|}{ Nafol } & linpar \\
\hline$s 1$ & 0.1763 & 0.2640 & 0.4355 & 1.7342 & 7.3151 & 90.0749 \\
\hline$s 2$ & 0.2083 & 0.7816 & 1.0235 & 0.1620 & 6.0365 & 91.7881 \\
\hline$s 3$ & 0.1553 & 1.2964 & 1.3756 & 1.2131 & 7.0820 & 88.8776 \\
\hline$s 4$ & 0.8518 & 0.1095 & 0.0542 & 1.3116 & 6.4309 & 91.2420 \\
\hline$s 5$ & 1.6505 & 0.0825 & 1.3674 & 1.7036 & 7.4678 & 87.7282 \\
\hline$s 6$ & 1.7450 & 1.4403 & 1.4450 & 0.5116 & 6.7454 & 88.1127 \\
\hline$s 7$ & 0.7210 & 1.0258 & 1.2970 & 0.1630 & 7.9987 & 88.7945 \\
\hline$s 8$ & 0.1077 & 0.3116 & 1.4594 & 0.5398 & 6.8491 & 90.7324 \\
\hline$s 9$ & 1.3567 & 1.0338 & 0.5706 & 2.0548 & 8.0137 & 86.9704 \\
\hline$s 10$ & 0.0671 & 1.0313 & 0.5586 & 1.1763 & 6.0765 & 91.0902 \\
\hline$s 11$ & 1.6715 & 0.5328 & 0.1982 & 1.6286 & 6.4622 & 89.5067 \\
\hline$s 12$ & 1.4958 & 1.3864 & 0.3861 & 1.6468 & 6.6220 & 88.4629 \\
\hline$s 13$ & 1.9875 & 0.1748 & 1.1500 & 1.2481 & 7.3893 & 88.0503 \\
\hline$s 14$ & 1.0245 & 1.2823 & 1.3557 & 0.9976 & 6.7430 & 88.5969 \\
\hline$s 15$ & 1.6513 & 0.5880 & 0.7405 & 0.0958 & 6.6366 & 90.2878 \\
\hline$s 16$ & 0.5523 & 0.0410 & 0.6537 & 0.1135 & 7.5665 & 91.0730 \\
\hline$s 17$ & 1.9826 & 0.1518 & 1.2132 & 1.3414 & 6.5969 & 88.7141 \\
\hline$s 18$ & 1.1525 & 1.1707 & 0.0357 & 0.3340 & 7.9382 & 89.3689 \\
\hline$s 19$ & 1.5698 & 0.9942 & 0.6833 & 0.5480 & 7.8198 & 88.3849 \\
\hline$s 20$ & 0.9602 & 0.6218 & 0.5642 & 1.2318 & 7.8489 & 88.7731 \\
\hline$s 21$ & 1.3318 & 0.7134 & 1.3070 & 0.6957 & 7.7591 & 88.1930 \\
\hline$s 22$ & 0.1857 & 0.6804 & 0.8192 & 1.3823 & 6.2905 & 90.6419 \\
\hline$s 23$ & 0.9326 & 0.8213 & 1.1156 & 0.8557 & 6.1147 & 90.1601 \\
\hline$s 24$ & 0.6387 & 0.8707 & 0.7292 & 0.1352 & 6.3864 & 91.2398 \\
\hline$s 25$ & 0.6138 & 0.3303 & 1.4033 & 1.2065 & 7.2254 & 89.2207 \\
\hline & & & & & & \\
\hline
\end{tabular}

\section{Results and Discussion}

Figure 2 shows the elemental analysis chromatograms of rolling oil components (base oil and additive) and possible heavy oil contaminants along with one of the synthetically prepared oil sample that contains all of these components.
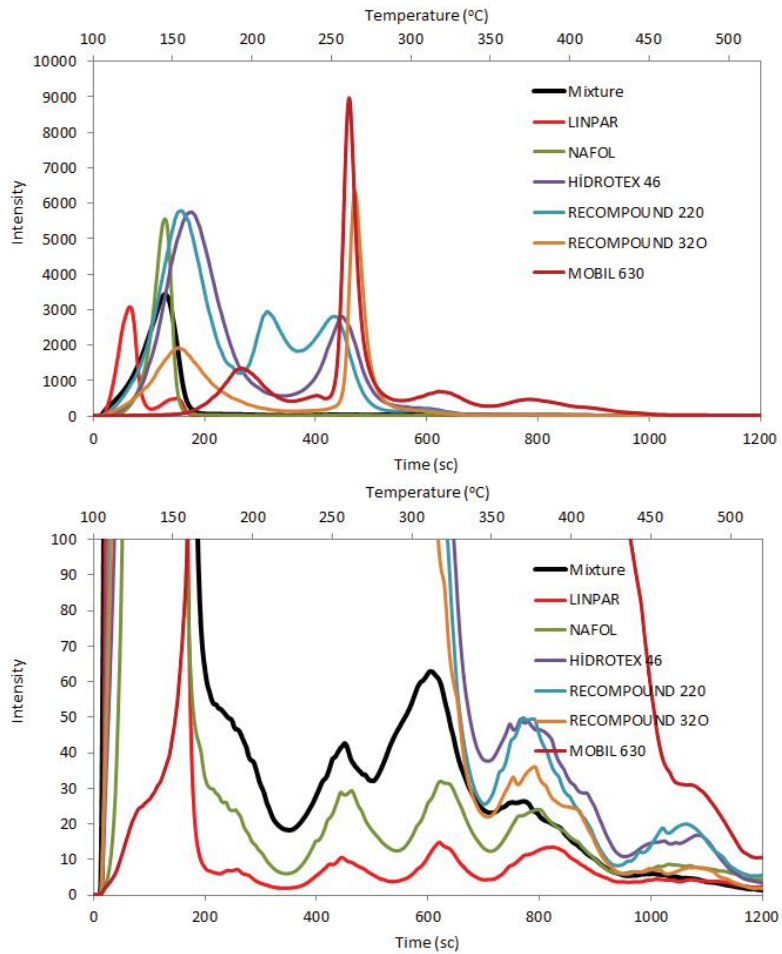

Figure 2. Carbon - Hydrogen elemental analysis chromatograms of rolling oil components (base oil and additive) and possible heavy oil contaminants along with a synthetically prepared mixture.
Although the primary horizontal axis is the time of the analysis, there are two horizontal axis on the top and at the bottom for both time and temperature scales of the temperature programming. The base oil of the rolling oil given in Figure 1 is the Linpar and the additive is Nafol which is given as red and green line, respectively. The heavy oil components are named as Hidrotex 46, Recompound 220, Recompound 320 and Mobil 630. The peaks that appear around the first 300 second are so intense that it would be imposible to see other peaks that are observed after 400 second thus the figure is also given in an enlarged scale at bottom. It is also worth to note that among the heavy oil components of Mobil 630 stronger peaks around 600 and 800 second whereas the other components show relatively low peaks beyond 400 seconds. Based on the temperature program, chromatograms shown in Figure 1 indicate a partial peak separation for different components but there are still strong overlaps and this would make difficult to quantify each component by means of univariate calibration. Throughout the multivariate calibration, it is expected to build quantitative models that would enable to predict individual component concentrations based on these weak differences seen on the chromatograms.

However, the ultimate goal in this particular study is to be able to predict the surface residual oil composition on the rolled aluminum sheet samples taken from the production. This requires that the chromatograms of the real samples must be comparable with the synthetically prepared sample chromatograms. Figure 3 shows the chromatograms of two rolled aluminum sheet samples with triplicate analysis from edges (right and left) and middle part of the sheets along with the base oil and one of the synthetic sample chromatogram.
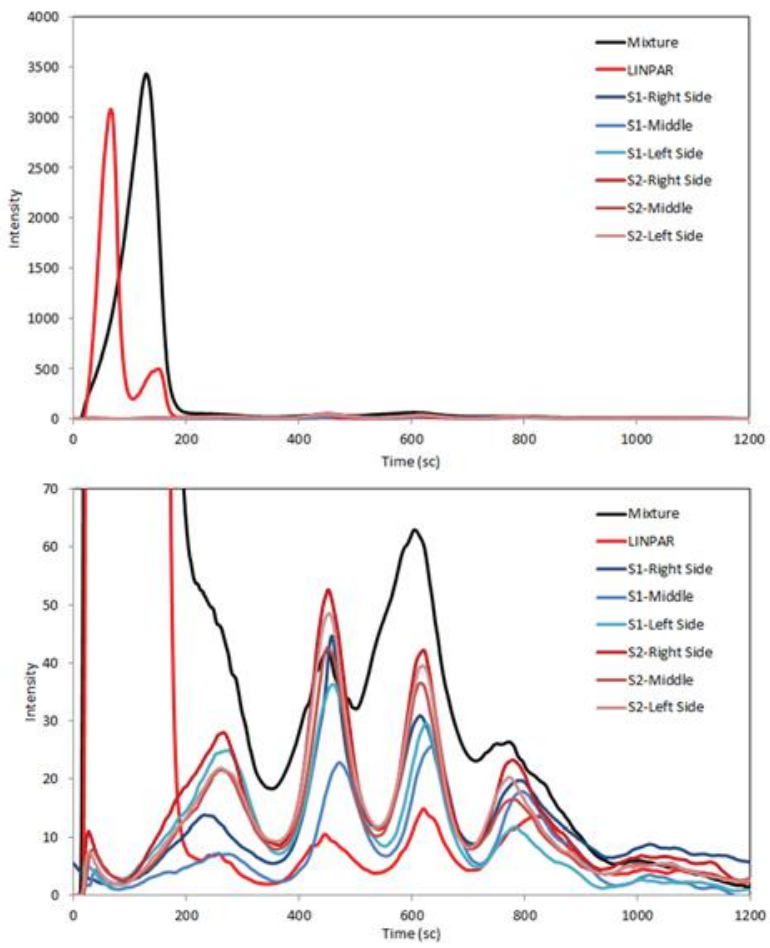

Figure 3. Carbon - Hydrogen elemental analysis chromatograms of base oil Linpar, one of the synthetic samples (Mixture) and two rolled aluminum sheet sample.

The most noticeable difference between rolled aluminum sheet chromatograms and the synthetic sample chromatogram is that the 
peak around the first $100 \mathrm{~s}$ is so dominating for the synthetic sample. It is almost impossible to see any feature on the chromatograms after 200 seconds in the rest of the chromatograms. This difference is due to the fact that the rolled aluminum sheets were thawed at temperatures above $300^{\circ} \mathrm{C}$ for a period of time after rolling process and therefore any base oil residue on the sheet samples are already removed from the surface of the sheets whereas synthetically prepared samples were analyzed as they are prepared by applying a tiny droplets of the oil mixture on the surface of aluminum. Yet, the enlarged view of the bottom chromatogram given in Figure 3 is still quite informative in terms of the homogeneity of the surface heavy oil residues. As can be seen from the enlarged bottom chromatogram rolled sheet sample named as $\mathrm{S} 1$ shows significant surface residue differences compared to sample named S2 in which the surface heavy oil residues were distributed in a somewhat more homogeneous manner from sides to the interior of the rolled sheet. Despite the significant differences in the first 200 second part of the chromatograms, the chromatographic profile of the synthetic sample is quite consistent with the rolled sheet samples which are promising for the future part of this study. Figure 4 shows the chromatograms of the 25 synthetic samples given in Table 1 .
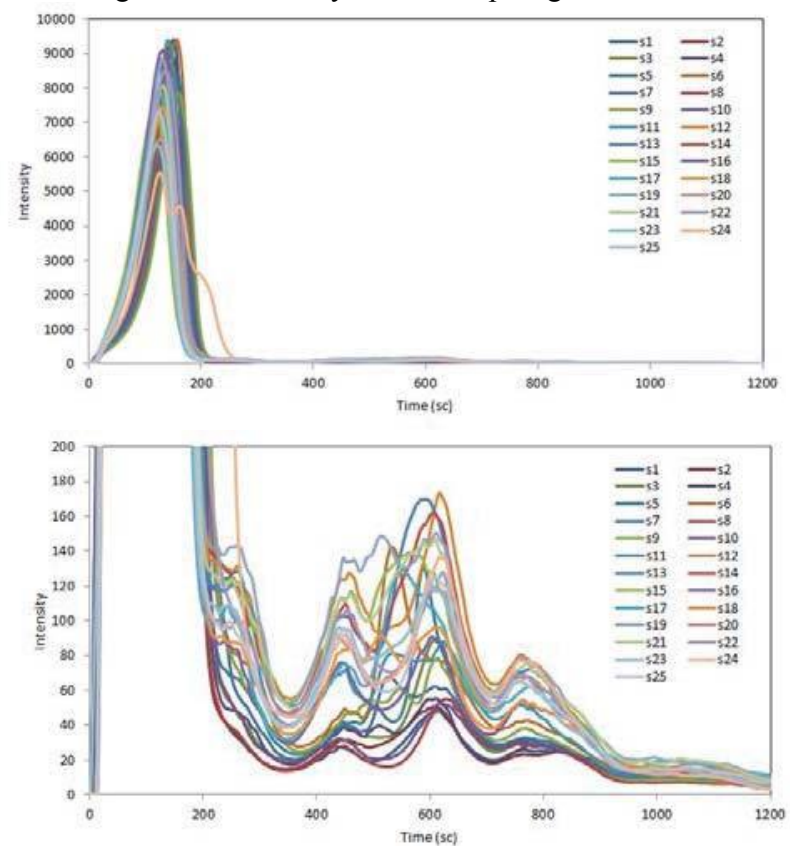

Figure 4. Carbon - Hydrogen elemental analysis chromatograms of the 25 synthetic samples (Mixtures) given in Table 1.

As the composition of the synthetic samples different in terms of the heavy oils given in Table 1, resulting chromatograms shows differences in chromatographic profiles not only in the first 200 second part but also in the further part of the chromatograms but the differences are more characteristic in the range from 200 to 1000 second. It is expected that these differences would provide a way to build quantitative multivariate calibration models for heavy oil components of the mixtures. In order to determine component concentrations of the synthetic samples with multivariate calibration models 18 of the samples given in Table 1 are randomly selected for calibration set and the remaining 7 samples were used to build independent validation set. A genetic algorithm based multivariate calibration method named genetic inverse least squares (GILS) is used to develop calibration models. Figure 5 shows the reference versus GILS predicted component concentrations for the four heavy oils contained in the synthetic mixtures.
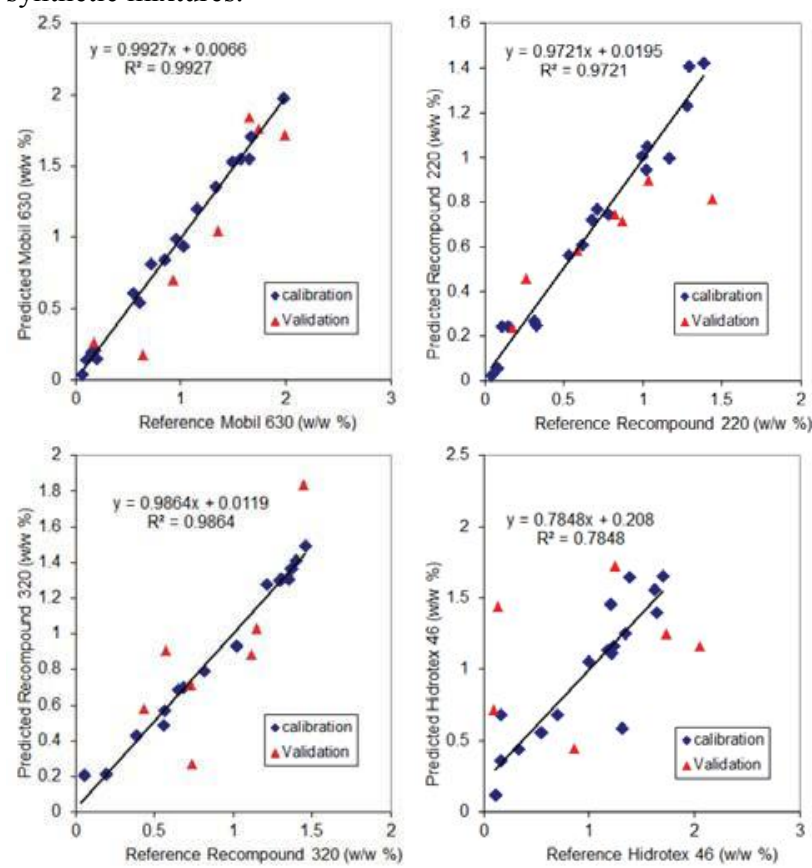

Figure 5. Reference vs GILS predicted concentration plots of heavy oil components.

As a preliminary study, there were only 25 synthetic samples and therefore there were a very limited number of samples to build calibration and independent validation sets. As can be seen in Figure 5, multivariate calibration models generated with GILS are quite good for the Mobil and Recompound components but poor for Hidrotex. On the other hand, the prediction results for the independent validation sets were not as good as the calibration sets. This might bring the question about over fitting of the models for the calibration samples, as it is based on an iterative variable selection method, so the models are unable to predict the validation samples. However this is not the case in this study because the GILS method used here relies on a leave one out cross validation to avoid overfitting during the model building and in fact the model for Hidrotex is not good at all. One of the most reasonable causes for the poor predictive ability of the models generated by GILS is the limited number of calibration samples which limits the number of selected variables on the full chromatograms as in ILS the number of variables cannot be more than the number of calibration samples. Nevertheless, as an initial study which is one of the first time in the literature for the multivariate application of elemental analysis, the current study demonstrate an interesting and difficult application on this method for the quantitative determination of rolling oil contaminants on the rolled aluminum sheets.

When compared to other components, it is seen that the model generated for Mobil 630 is much better for the predictive ability and this is not surprising as it is the most dominating components among the others in the chromatograms given in Figure 2. In fact, Hidrotex is the weakest component in the chromatograms in terms of peak intensities which means that it has a higher tendency to volatilize at lower temperatures when compared to other heavy oils. The standard error of the calibration (SEC) and standard error of prediction (SEP) for the calibration set and independent 
validation set for Mobil were $0.05(\mathrm{w} / \mathrm{w} \%)$ and $0.26(\mathrm{w} / \mathrm{w} \%)$ whereas the Recompound 220 and 320 these values were ranged from 0.06 to $0.29(\mathrm{w} / \mathrm{w} \%)$ for both calibration and validation sets respectively. For Hidrotex, while SEC value was around 0.25 (w/w \%), the SEP value was much higher $(0.89 \mathrm{w} / \mathrm{w} \%)$ showing that the model was unable predict the validation samples. As a result of the strong overlaps in the chromatograms the GILS method was having difficulty to establish robust calibration models and this could be confirmed if one decides to build total heavy oil content of the samples but summing their individual concentrations. Figure 6 shows the results of these models in which first all of the four heavy oil concentrations are added and then this is done without Hidrotex since it the one which GILS had difficulty to develop model.
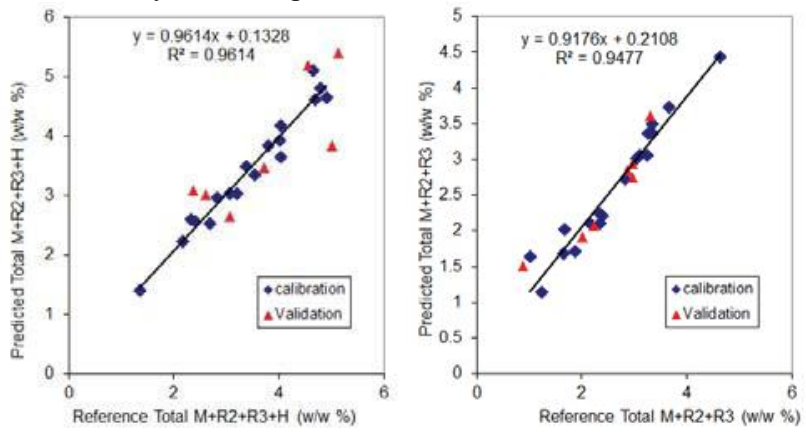

Figure 6. Reference vs GILS predicted plots of total contaminants models.

As seen on the Figure 6, when Hidrotex is not used in the total contaminant modeling a much better model is developed in terms of predictive ability of the model compared to the case where all of the four components used. Because the GILS method is based on the a genetic algorithms for the variable selection it is worth to see when the algorithm is repeated for predefined times and generate frequency distribution of the selected point on the chromatograms. Figure 7 shows frequency distribution plot of GILS models when the algorithm was set to run 100 times.
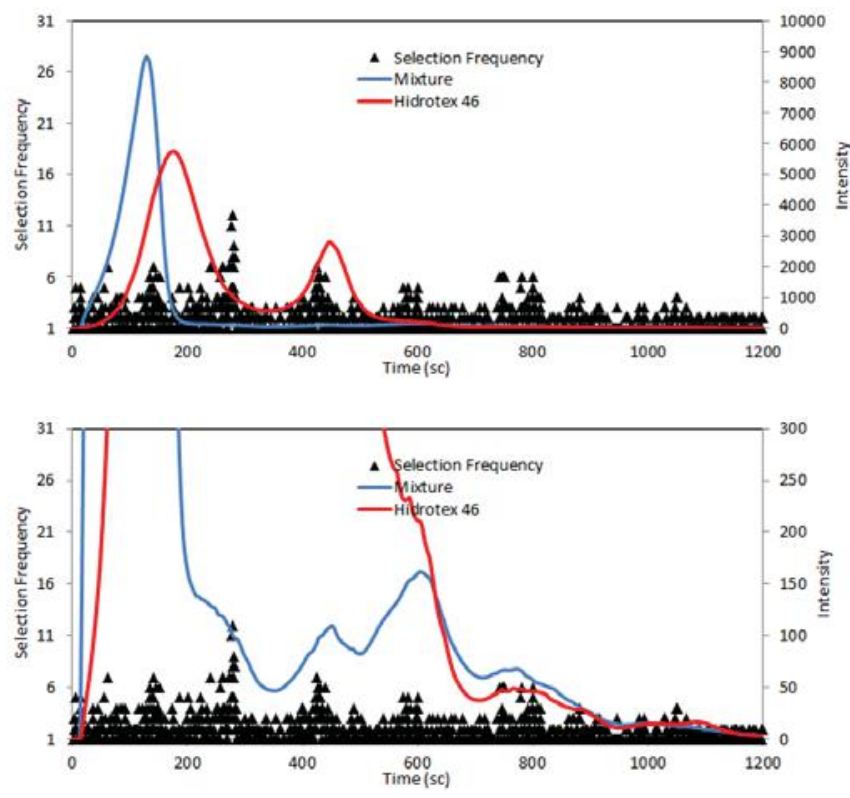

Figure 7. Selection frequency plot of the selected variables by GILS.
As seen on the Figure 7, the GILS method aims to focus on the variables that are more relevant to the component of the interest which indicates that the method concentrates the regions where component information is located. This frequency plot is only generated as the models for the other components did not show the same predictive ability for the independent validation set. Even though the predictive ability of the models were somewhat poor for most of the component except the Mobil 630, this study demonstrated that with more samples in the calibration sets elemental analysis with proper temperature programming could be used to quantify heavy oil components on the aluminum sheets.

Acknowledgment: Authors wish to thank Mr. Hikmet Kayaçetin, technician of Assan Aluminum Laboratory, for his meticulous work in preparing samples.

\section{References}

1. A. Yalçın, D, Ergun, Ö.İ. Uçar, and D. Özdemir, "Determination of Aluminum Rolling Oil Additives And Contaminants Using Infrared Spectroscopy Coupled With Genetic Algorithm Based Multivariate Calibration", Vibrational Spectroscopy 54, 10-20 (2010).

2. J.A. Lopesa, P. F. Costaa, T. P. Alvesb, and J. C. Menezes. "Chemometrics in Bioprocess Engineering: Process Analytical Technology (PAT) Applications", Chemometrics and Intelligent Laboratory Systems, 74, 269 (2004).

3. P. Geladi and B.R. Kowalski, "Partial Least Squares Regression: A Tutorial", Anal. Chim. Acta., 185, 1 (1986).

4. D.M. Haaland, and E.V. Thomas, "Partial Least Squares Methods for Spectral Analyses. Relation to Other Quantitative Calibration Methods and the Extraction of Qualitative Information”. Anal. Chem., 60, 1193 (1988).

5. P.D. Wentzell, D.T. Andrews, and B.R. Kowalski, "Maximum Likelihood Multivariate Calibration", Anal. Chem., 69, 2299 (1997).

6. C.B. Lucasius, M.L.M. Beckers, and G. Kateman, "Genetic Algorithms in Wavelength Selection : A Comparative Study”, Analytica Chimica Acta, 286(2), 135 (1994)

7. U. Hörchner, and J.H. Kalivas, "Further Investigation on a Comparative Study of Simulated Annealing and Genetic Algorithm for Wavelength Selection", Anal. Chim. Acta., 311, 1 (1995).

8. R. Leardi, "Application of Genetic Algorithm-PLS for feature selection in spectral data sets", J. Chemometrics, 14,643 (2000).

9. H. C.Goicoechea1 and A. C.Olivieri, "A New Family of Genetic Algorithms for Wavelength Interval Selection in Multivariate Analytical Spectroscopy", J. Chemometrics, 17, 338 (2003).

10. J. Koljonen, T.E.M. Nordling, and J.T. Alander, "A Review of Genetic Algorithms in Near Infrared Spectroscopy and Chemometrics: Past and Future", Journal of Near Infrared Spectroscopy, 16(3), 189 (2008).

11. C. Reynès, S. de Souza, R.t Sabatier, G. Figuères, and B. Vidal, "Selection of Discriminant Wavelength Intervals in NIR Spectrometry with Genetic Algorithms", J. Chemometrics, 20, 136 (2006). 
12. D. Özdemir, and B. Öztürk, "Genetic Multivariate Calibration Methods for Near Infrared (NIR) Spectroscopic Determination of Complex Mixtures", Turk. J. Chem., 28, 497 (2004).

13. D. Özdemir, and B. Öztürk, "Near Infrared Spectroscopic Determination of Olive Oil Adulteration with Sunflower and Corn Oil", Journal of Food and Drug Analysis, 15(1), 40 (2007).

14. B. Uner, İ. Karaman, H. Tanriverdi, and D. Özdemir, "Prediction of Lignin and Extractive Content of Pinus nigra Arnold. var. Pallasiana Tree Using Near Infrared Spectroscopy and Multivariate Calibration", Journal of Wood Chemistry and Technology, 29, 24 (2009). 